\title{
Correction
}

\section{Correction to: Interseismic Coupling and Slow Slip Events on the Cascadia Megathrust}

\author{
Sylvain Michel, ${ }^{1,2}$ (D) Adriano Gualandi, ${ }^{2,3}$ and Jean-PhilipPe Avouac ${ }^{2}$
}

Correction to: Pure Appl. Geophys.

https://doi.org/10.1007/s00024-018-1991-x

Owing to an unfortunate oversight, the wrong figure 2 has been published. Unfortunately also the acknowledgement has been omitted.

\section{Acknowledgements}

We thank Kelin Wang for his constructive review of this manuscript. This study was partially supported by NSF award EAR-1821853 to JPA. We thank also Zachary E. Ross for his help. We thank Richard Walters and David Al-Attar for their detailed and constructive criticism which helped improve our study.

The authors apologize for this mistake.

The original article can be found online at https://doi.org/10.1007/ s00024-018-1991-x.

1 Bullard Laboratories, Department of Earth Sciences, University of Cambridge, Madingley Road, Cambridge, Cambridgeshire CB3 0EZ, UK. E-mail: sylvain_michel@live.fr

2 Department of Geology and Planetary Sciences, California Institute of Technology, 1200 E California Blvd, Pasadena, CA 91125, USA. E-mail: adriano.geolandi@gmail.com; avouac@gps.caltech.edu

3 Jet Propulsion Laboratory, California Institute of Technology, 4800 Oak Grove Dr, Pasadena, CA 91109, USA. 

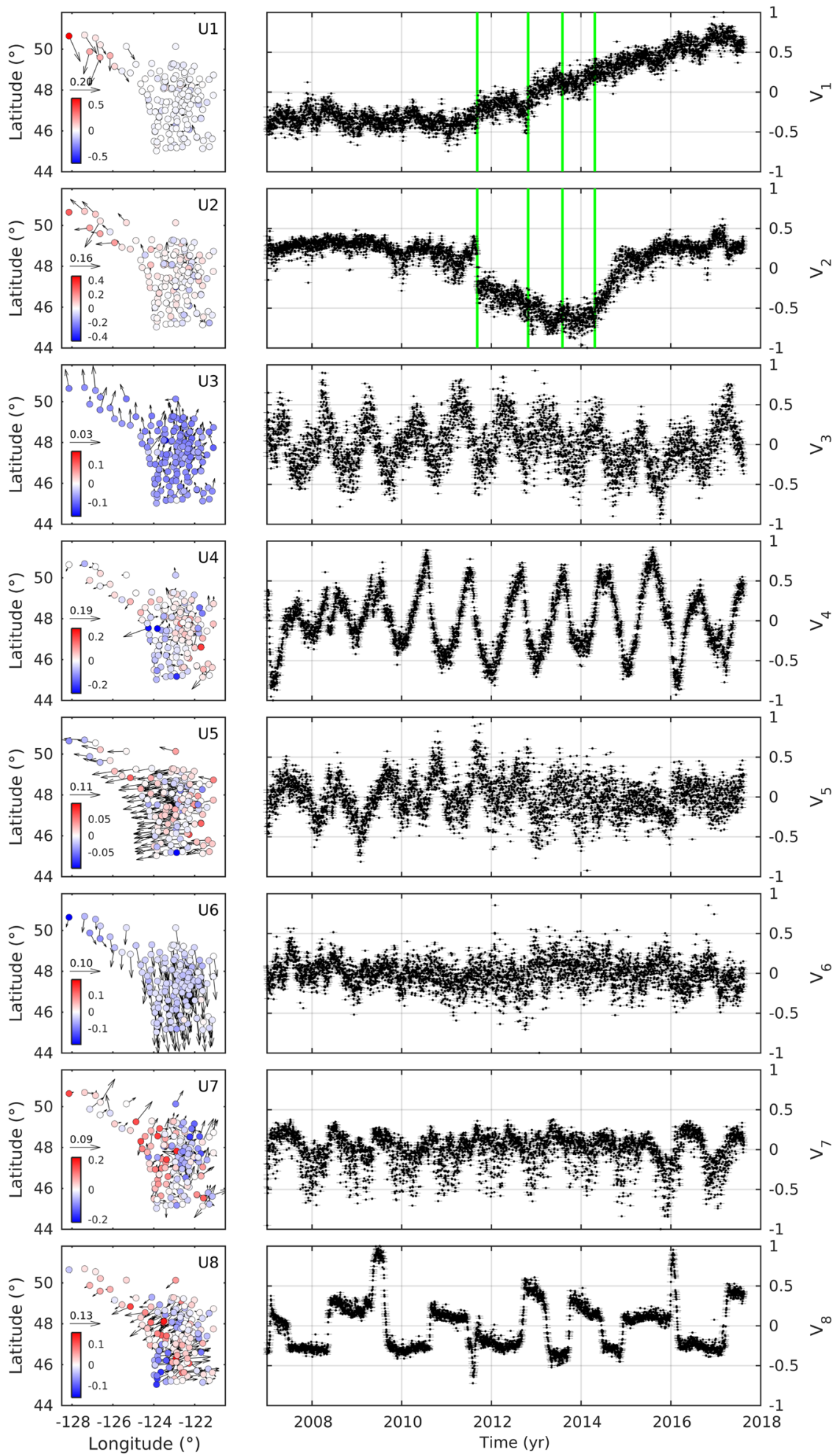

(Published online October 31, 2018) 